\title{
Monitoring the failure states of a metal matrix syntactic foam by
}

\section{modal analysis}

\author{
Csilla Kádár ${ }^{1,2 *}$, Attila Szlancsik ${ }^{1,2}$, Zoltán Dombóvári $^{2,3}$, Imre Norbert Orbulov $^{1,2}$ \\ ${ }^{1}$ Department of Materials Science and Engineering, Budapest University of Technology \\ and Economics, Müegyetem rkp. 3., H-1111 Budapest, Hungary \\ ${ }^{2}$ MTA-BME Lendület Composite Metal Foams Research Group, Mủegyetem rkp. 3.,H- \\ 1111, Budapest, Hungary \\ ${ }^{3}$ Department of Applied Mechanics, Budapest University of Technology and Economics, \\ Mủegyetem rkp. 3., H-1111 Budapest, Hungary
}

Keywords: Porous materials, Deformation and fracture, Modal analysis, Syntactic foams

\begin{abstract}
This paper presents a new non-destructive method applying modal analysis to determine the failure state of theAlSi12 matrix syntactic foam containing ceramic hollow spheres. The results proved that the decrease in natural frequencies of the first two modes and the calculated effective Young's modulus indicate the damage state of the syntactic foam's structure during compression. Both the natural frequencies and the elastic modulus start to change after the onset of fracture of hollow spheres, which is $\sim 60 \mathrm{MPa}$, according to our

${ }^{*}$ Corresponding author. E-mail addresses: kadar@bme.eik.hu (Cs. Kádár),
\end{abstract}


previous work. A 5\% decrease in these parameters are suggested as a practical limit of the deterioration of a load-bearing structure, which collides with the maximum in the acoustic emission activity.

\section{Introduction}

Metal matrix syntactic foams (MMSFs) have been brought to attention due to their good mechanical performance. They are light and strong, have high energy absorption capacity, therefore they are ideal for load bearing and energy absorbers structures [1].

Non-destructive methods for diagnosing engineering structures are beneficial and sometimes crucial to guarantee the desired quality [2]. The non-destructive methods, which have been applied for monitoring the failure mechanisms are either expensive or time consuming (e.g. X-ray tomography). Acoustic emission (AE) is a better solution, since it is cheap and provides information on the failure mechanisms of the entire volume of the sample. Since AE is reliable for the identification of failure mechanisms during deformation, the sample must be loaded during the measurement.

Modal analysis (MA) has been proved to be an effective non-destructive technique to measure vibration properties of engineering structures [3]. Even though MA is strictly a linear technique in principle, it is capable of detecting critical defects by monitoring the changes in the vibration properties [3-5], since structural damage of the material results in degradation of stiffness and can cause drops in the dominant natural frequencies.

Our goal was to establish a cheap and simple non-destructive method to characterize the damage states of unloaded MMSFs, so the accumulation of the defects can be monitored 
even in long-term deformation processes (e.g. fatigue). Our focus was to find parameters measured by MA that the first sign of integrity degradation, and later the complete fracture state can be predicted.

\section{Background}

Previous investigations revealed the failure mechanisms during compression in MMSFs with AlSi12 matrix, reinforced by approximately 50 vol\% Globocer ${ }^{\circledR}$ hollow ceramic spheres [6]. The AE measurements and their evaluation by adaptive sequential k-means (ASK) clustering technique revealed that the two failure mechanisms occurring already in the quasi-linear stage $(<50 \mathrm{MPa})$ are the plastic deformation and the fracture of the brittle phases (Al-Si-Fe intermetallic and Si phases) in the matrix. It was found that the onset of the fracture of the spheres is approximately $\sigma_{\mathrm{SFH}}=60 \mathrm{MPa}$, regardless of the matrix material [6]. The AE response is maximum before the stress reaches its peak at $\sim 80 \mathrm{MPa}$, which is approximately $70 \%$ of the maximum stress value (Fig. 1a). Note that at this stage of the deformation the digital image correlation technique has not revealed considerable strain localization on the surface.
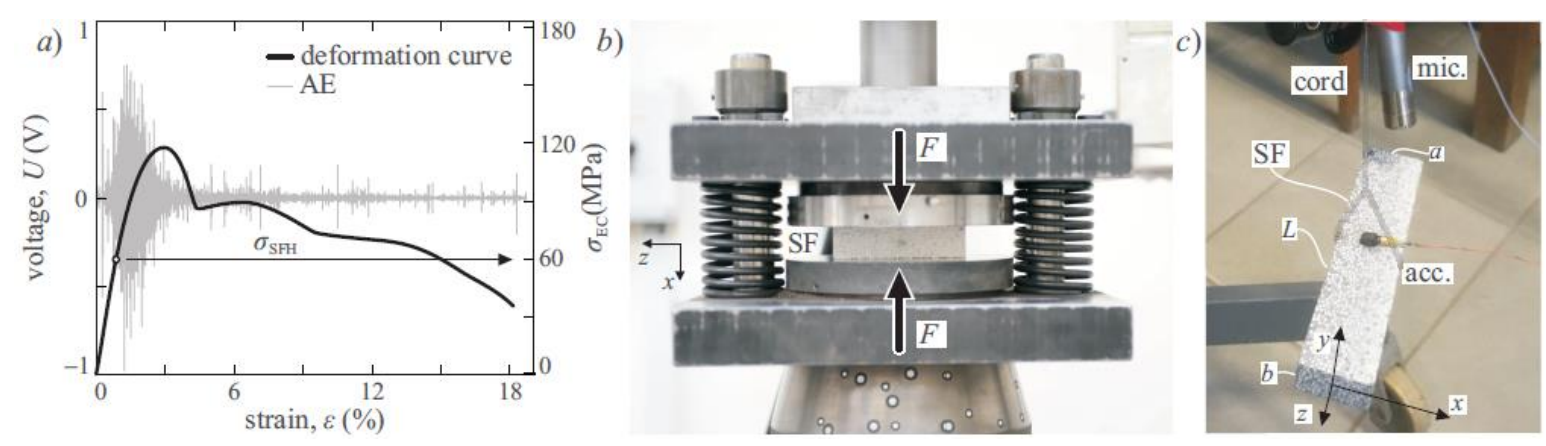
Figure 1. (a) Stress-strain curve with the corresponding AE response [6]. The onset of fracture of hollow spheres is indicated by $\sigma_{\mathrm{SFH}}$. The arrangement of the compression test (b) and the MA tests (c).

A measurement technique based on homogenized Timoshenko beam theory using experimental MA was developed and verified for the same MMSF [7]. The MA measurement was performed with impulse excitation [7] and the response was acquired with a microphone. The specimens in [7] were modified to have a flat surface performing 'perfect' hits with good coherence [3].

\section{Experimental}

In this paper the same type of MMSF with AlSi12 matrix was investigated as in [6,7] produced by the manufacture process of [8].

We used an MTS 810 universal testing machine for the compression test on samples of cross-section $100 \times 15 \mathrm{~mm}$ and height $25 \mathrm{~mm}$. The compression was performed at room temperature in force control of $0.03 \mathrm{~N} / \mathrm{s}$ force rate in a four-column equipment to ensure uniaxial loading (Figure 1b). The specimen was wound around by a thin teflon tape to minimize friction.

The sample was loaded to a certain force level and then unloaded 37 times as detailed below. In each cycle, after unloading, the size of the specimen was measured, then MA was performed (on the already deformed sample), and also a picture of the deformed surface 
was taken. The load increment was $3 \mathrm{kN}$ at the beginning of deformation. After reaching $30 \mathrm{kN}$ the load increment was increased to $5 \mathrm{kN}$.

MA tests were performed on the same unloaded block of foam hanging on an elastic rubber cord (Fig. 1c). This support situation is the closest to the free-free condition, which can be modelled quite precisely by Timoshenko beam theory. This kind of hanging arrangement also provides the smallest damping (less contact - less damping). Besides the microphone used in the measurement presented in [7], we also used an accelerometer sensor with its cord stripped in order to decrease its effect on damping and stiffness. The accelerometer sensor was placed in the middle of the sample (Fig. 1c) in order to have the balanced inertial effect on both bending modes [3]. Four impact excitations were performed in each cycle and direction ( $x$ and $y$ ) at one side of the sample by a micro impulse hammer [3]. Since the specimen did not have flat surfaces to perform a 'perfect' hit, the results of the three best impulse excitations (i.e. showing highest coherence) were used for the evaluation. The natural frequencies $\left(\omega_{\mathrm{n} 1}, \omega_{\mathrm{n} 2}\right)$ were manually read from the graph of the measured frequency response functions. The damping $(\zeta)$ and the effective Young's modulus (E) were calculated by the techniques in [3] and [9], respectively.

\section{Results and discussion}

A total of 37 loading-unloading cycles were performed until the collapse of the specimen appeared in the 38 th cycle at the peak (120MPa) of the stress-strain curve (Fig. 1a). The surface pictures in the first 37 cycle had not shown any sign of crack, however, some missing ceramic spheres were visible after the stress exceeded $80 \mathrm{MPa}$. 
Fig. 2 shows the different parameters obtained by MA measurements. The relative changes in the second moment of inertias (Fig. 2a) show the effect of changes in geometry due to compression. Changes in $I_{x}$ and $I_{y}$ become significant only close to the complete failure of the foam, showing that geometrical parameters are not effective in predicting failure in advance. Note that in MMSFs used as a load-bearing structure the degradation of the material properties must be avoided long before the final failure.
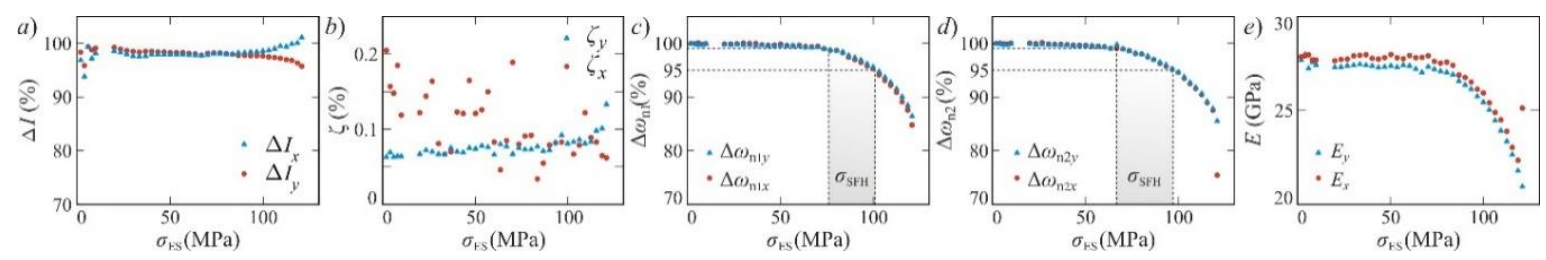

Figure 2. Relative changes in second moment of inertia (a), damping (b), and natural frequencies in mode one (c) and two (d) w.r.t. applied engineering stress $\sigma_{\mathrm{ES}}$. Each parameter was measured by MA for the $x$ and $y$ bending axes (Fig. 1c). Grey shading shows stress intervals from the onset of fracture of hollow spheres $\left(\sigma_{\mathrm{SFH}}\right)$ to the $5 \%$ practical limit of the degradation in natural frequencies. The effective Young's modulus calculated by Timoshenko beam theory using the first two modes $(\mathrm{e}) .\left(\Delta \bullet\left(\sigma_{\mathrm{ES}}\right):=\bullet\left(\sigma_{\mathrm{ES}}\right) / \bullet(0)\right.$, where denotes an arbitrary parameter (e.g. $I, \zeta), \bullet(0)$ and $\bullet\left(\sigma_{\mathrm{ES}}\right)$ are the values of the parameter prior to the deformation and at a given $\sigma_{\mathrm{ES}}$ stress, respectively.)

It was expected that the onset of fracture of hollow spheres determined by the ASK evaluation on the $\mathrm{AE}$ data can be seen on the modal damping characteristics due to the increased internal friction, caused by the fractured ceramic sphere pieces. However, as it 
can be seen in Fig. 2b, the values of the modal damping of the first bending mode in the case of $x$ directional excitation $\left(\zeta_{1 x}\right)$ scatter. Even in the $y$ directional excitation, where the scatter is negligible, only a moderately increasing tendency can be recognized directly before the final failure, similar to Fig. 2a. This shows that modal damping is not suitable to predict the onset of fracture of hollow spheres.

The changes in the first and second natural frequencies $\left(\omega_{\mathrm{n} 1}, \omega_{\mathrm{n} 2}\right)$ can be seen in Figs. $2 \mathrm{c}$ and $2 \mathrm{~d}$. The curvature of the curves in Fig. $2 \mathrm{c}$ and $2 \mathrm{~d}$ start to increase drastically at $65 \mathrm{MPa}$ and $75 \mathrm{MPa}$, respectively. This point corresponds to less than $1 \%$ degradation in natural frequencies. This is obviously not a good practical limit, it is advisable to choose $5 \%$ instead, which still significantly precedes the collapse of the material (120 MPa).

The effective Young's modulus for both $x$ and $y$ direction excitations as the function of stress show similar tendencies as the bending natural frequencies (Fig. 2d). The syntactic foam specimen had a rectangular cross section, which means that the effective Young's modulus can be calculated by both $x$ and $y$ excitations, separately. The agreement in Fig. 2e is remarkable, indicating that the quality of the MA measurement is adequate.

\section{Conclusion}

The MA during compression of a MMSF was investigated in this work. The results proved that the decrease of the natural frequencies of the first two bending modes corresponds to the onset of fracturing of the hollow spheres determined previously by AE. Setting a decrease of $5 \%$ of the initial natural frequencies, MA can be used as a predictive maintenance technique for detecting damages. This $5 \%$ decrease is significantly before the 
damage can be detected on the surface of the sample or by the changes in the geometric properties.

\section{Acknowledgement}

This paper was supported by the János Bolyai Research Scholarship of the Hungarian Academy of Sciences (Cs. Kádár ), the Higher Education Excellence Program of the Ministry of Human Capacities in the frame of Nanotechnology research area of Budapest University of Technology and Economics (BME FIKP-NAT) and the National Research, Development and Innovation Fund (TUDFO/51757/2019-ITM, Thematic Excellence Program).

\section{References}

[1] M.F. Ashby, A.G. Evans, N.A. Fleck, L.J. Gibson, J.W. Hutchinson, H.N.G. Wadley, Metal Foams: A Design Guide, Butter-worth-Heinemann, Boston, 2000.

[2] M Musiał, J Grosel, Determining the Young's modulus of concrete by measuring the eigenfrequencies of concrete and reinforced concrete beams, Constr. Build. Mater. 121 (2016) 44-52. https://doi.org/10.1016/j.conbuildmat.2016.05.150

[3] Ewins DJ (2000) Modal Testing, Theory, Practice, \& Application, 2nd edition. Wiley, Hoboken, USA. 
[4] C.M. Newtson, G.P. Johnson; B.T. Enomoto, Fundamental Frequency Testing of Reinforced Concrete Beams, J Perform. Constr. Facil. 20 (2006) 196-200. https://doi.org/10.1061/(ASCE)0887-3828(2006)20:2(196)

[5] M. Krawczuk., W. Ostachowicz, A. Zak, Modal analysis of cracked, unidirectional composite beam, Compos. Part B-Eng., 28 (1997) 641-650. https://doi:10.1016/s1359$\underline{8368(97) 82238-x}$

[6] Cs. Kádár, K. Máthis, M. Knapek, F. Chmelík, The Effect of Matrix Composition on the Deformation and Failure Mechanisms in Metal Matrix Syntactic Foams during Compression, Materials 10(2) (2017) 196. https://doi.org/10.3390/ma10020196.

[7] A. Szlancsik, B. Katona, Z. Dombóvári, I.N. Orbulov, On the effective Young’s modulus of metal matrix syntactic foams, Mater. Sci. Tech. 33 (2017) 2283-2289. https://doi.org/10.1080/02670836.2017.1374497

[8] I.N. Orbulov, J. Ginsztler, Comressive behaviour of metal matrix syntactic foams, Acta Polytech. Hung., 9 (2012) 43-56.

[9] L. Majkut, Free and forced vibrations of Timoshenko beams described by single difference equation, J. Theor. App. Mech-Pol.,47 (2009) 193-210.

\section{Figure Captions}

Figure 1. (a) Stress-strain curve with the corresponding AE response [6]. The onset of fracture of hollow spheres is indicated by $\sigma_{\mathrm{SFH}}$. The arrangement of the compression test (b) and the MA tests (c). 
Figure 2. Relative changes in second moment of inertia (a), damping (b), and natural frequencies in mode one (c) and two (d) w.r.t. applied engineering stress $\sigma_{\mathrm{ES}}$. Each parameter was measured by MA for the $x$ and $y$ bending axes (Fig. 1c). Grey shading shows stress intervals from the onset of fracture of hollow spheres $\left(\sigma_{\mathrm{SFH}}\right)$ to the $5 \%$ practical limit of the degradation in natural frequencies. The effective Young's modulus calculated by Timoshenko beam theory using the first two modes (e). $\left(\Delta \bullet\left(\sigma_{\mathrm{ES}}\right):=\diamond\left(\sigma_{\mathrm{ES}}\right) / \diamond(0)\right.$, where denotes an arbitrary parameter (e.g. $I, \zeta),(0)$ and $\diamond\left(\sigma_{\mathrm{ES}}\right)$ are the values of the parameter prior to the deformation and at a given $\sigma_{\mathrm{ES}}$ stress, respectively.) 